\title{
Bilateral PHEOCHROMOCYTOMA/INTRA-ADRENAL PARAGANGLIOMA IN VON HIPPEL-LINDAU PATIENT CAUSING ACUTE MYOCARDIAL INFARCTION
}

\author{
Svitlana Bachurska ${ }^{1}$, Dmitriy Staykov ${ }^{1}$, Veselin Belovezhdov ${ }^{1}$, Hironobu Sasano ${ }^{2}$, \\ Milena Gulinac ${ }^{1}$, Chavdar Stefanov ${ }^{3}$, Hartmut P.H. Neumann ${ }^{4}$
}

\author{
${ }^{1}$ Department of General and Clinical Pathology, Medical University of Plovdiv, University Hospital “St. George”, Plovdiv, \\ Bulgaria \\ ${ }^{2}$ Department of Pathology, Tohoku University School of Medicine, Tohoku University Hospital, Sendai, Japan \\ ${ }^{3}$ Department of Anesthesiology and Intensive Care, Medical University of Plovdiv, University Hospital "St. George", \\ Plovdiv, Bulgaria \\ ${ }^{4}$ Section for Preventive Medicine, Department of Nephrology and General Medicine, University Medical Center, \\ Albert-Ludwigs-University of Freiburg, Freiburg, Germany
}

\begin{abstract}
A 26-year-old male presented to the emergency department complaining of obstipation, severe headache and abdominal pain. An autopsy revealed bilateral pheochromocytoma and acute myocardial infarction. The tumor cells showed positive immunoreactivity of both chromogranin A and synaptophysin and were negative for adrenocortical markers such as SF-1, c17, scc, 3 $\beta$-HSD as well as SDHB, suggesting a germline mutation of the gene SDHB or SDHD. Molecular genetic analyses did not show a mutation in these two genes, but a mutation in the VHL gene, in exon 3: VHL c.499C $>$ T. This is a missense mutation and causes an amino acid change (Arg167Trp).
\end{abstract}

Key words: von Hippel-Lindau disease, pheochromocytoma, myocardial infarction, autopsy.

\section{Introduction}

Von Hippel-Lindau (VHL) disease is an autosomal dominant disorder associated with the development of prototypic lesions such as retinal angiomas, hemangioblastomas of the central nervous system, epididymal cystadenomas, renal cysts and renal cell carcinomas, pheochromocytomas, and pancreatic islet cell tumors [1]. Pheochromocytomas and paragangliomas (extra-adrenal pheochromocytoma) are catecholamine-producing neuroendocrine tumors arising from adrenal medulla and/or extra-adrenal chromaffin tissue. Not all paragangliomas are endocrinologically active and the classical clinical triad of headache, palpitations and sweating is only clinically detected in two thirds of affected patients $[2,3]$. Some patients are completely asymptomatic $[1,3]$ or present merely with non-specific clinical symptoms such as hyperglycemia $[3,4]$, acute abdomen $[3,5]$, neurological findings such as stroke [3] and cardiovascular events such as acute cardiovascular shock and myocardial infarction [3,7]. Therefore, despite improved diagnostic methods, many of the patients may still remain clinically undiagnosed $[7,8]$. In this case report, we describe an autopsy case of bilateral pheochromocytoma occurring in a young VHL male patient presenting as acute abdomen and resulting in acute myocardial infarction and multiorgan failure in his clinical course, leading, because of young age and 
bilateral adrenal involvement, to the question whether there may be an underlying inherited syndrome.

\section{Material and methods}

We present a case with a pheochromocytoma detected by autopsy. Molecular genetic analyses for germline mutations of the VHL gene were performed after DNA extraction from normal liver tissue using standard methods. All exons of the genes $S D H B$, SDHD, VHL and exons 10, 11 and 16 of the RET gene were sequenced (Sanger sequencing) after PCR amplification.

\section{Results}

The index case is a 26-year-old male with an uneventful previous medical history. The patient is an orphan; he used to live in very poor social conditions and never visited a family doctor. The family background of the parents and siblings history is unavailable. He presented to the emergency department for obstipation for the last four days, abdominal pain and general weakness which lasted a few hours prior to his visit. Physical examination revealed blood pressure of 160/100 mm Hg and heart rate of 120 beat per minute. Laboratory results and chest X-ray were non-contributory. The abdomen was tender and suggestive of acute peritoneal stimulation. Emergency surgery was performed because of paralytic ileus and peritonitis was clinically suspected. The subsequent laparotomy revealed air dilated intestinal loops and hepatomegaly. Surgery could not be continued further due to severe hemodynamic instability of the patient. Therefore the patient was transferred to the ICU and despite a combination of pressors and inotropes for the next 48 hours he developed severe depression of left ventricle function and elevated levels of creatine kinase, creatine kinase $\mathrm{MB}$ fraction and troponin. The

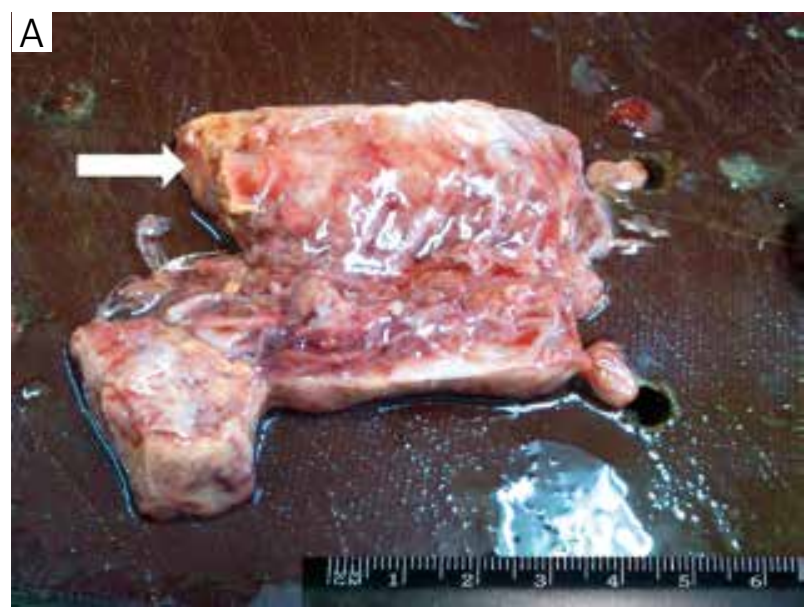

chest CT scan without contrast demonstrated bilateral pleural effusions, hypoventilated lungs and an enlarged heart. The abdominal CT scan with contrast revealed a right $6.2 \times 4.3 \mathrm{~cm}$ and a left $5.0 \times 4.0$ adrenal mass (Fig. 1). Bilateral pheochromocytoma or cortical adrenal adenoma was radiologically suspected. The patient subsequently died with signs of acute cardiogenic shock and multiorgan failure.

\section{Autopsy and molecular genetic findings}

Bilateral adrenal lesions were detected: $6.0 \times 5.0$ $\times 4.0 \mathrm{~cm}$ in the right (Fig. $2 \mathrm{~A}$ ) and $5.0 \times 4.0 \times$ $4.0 \mathrm{~cm}$ in the left suprarenal areas (Fig. 2B). Both were encapsulated, well circumscribed and appeared yellow admixed with scattered foci of hemorrhagic areas on their cut surface. Histologically, the right and left lesions were identical, the tumor cells having irregular shaped, large and hyperchromic nuclei with prominent nucleoli forming nests and cords supported by highly vasculated stroma (Fig. 3A,B). The heart $(570 \mathrm{~g})$ demonstrated moderate biventric-

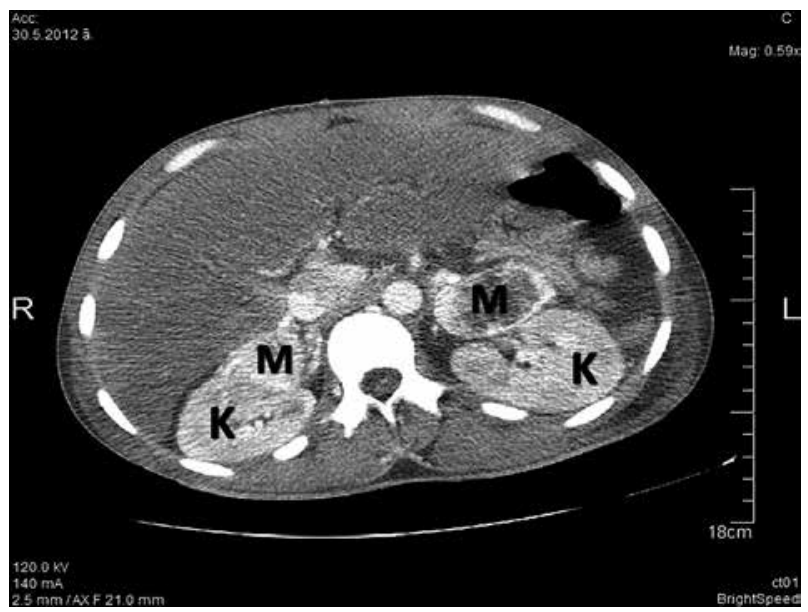

Fig. 1. The abdomen CT scan showing the bilateral suprarenal masses without signs of invasion ( $\mathrm{K}-$ kidney, $\mathrm{M}-$ mass)

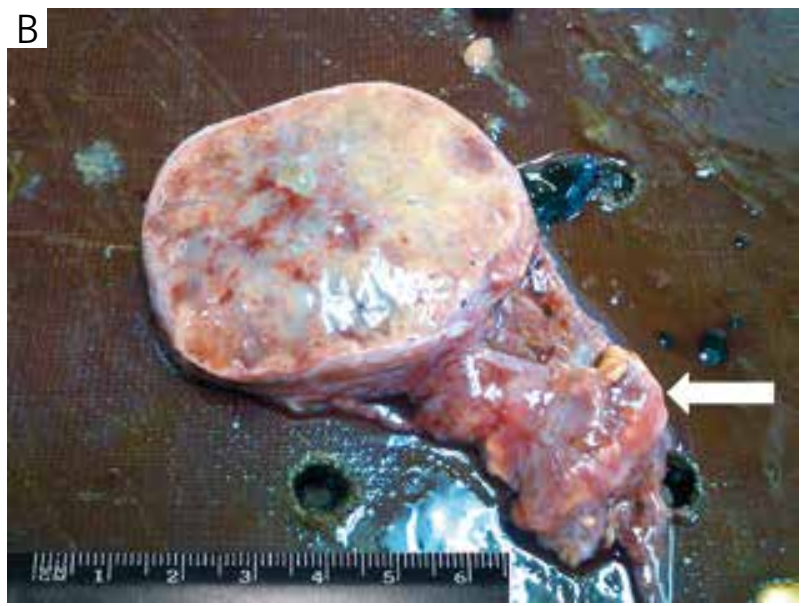

Fig. 2. A) Right intra-adrenal paraganglioma with part of normal adrenal cortex $(\rightarrow)$. B) Left tumor presenting well-circumscribed central pink-yellow with areas of hemorrhage intra-adrenal paraganglioma and part of normal adrenal cortex $(\leftarrow)$ 

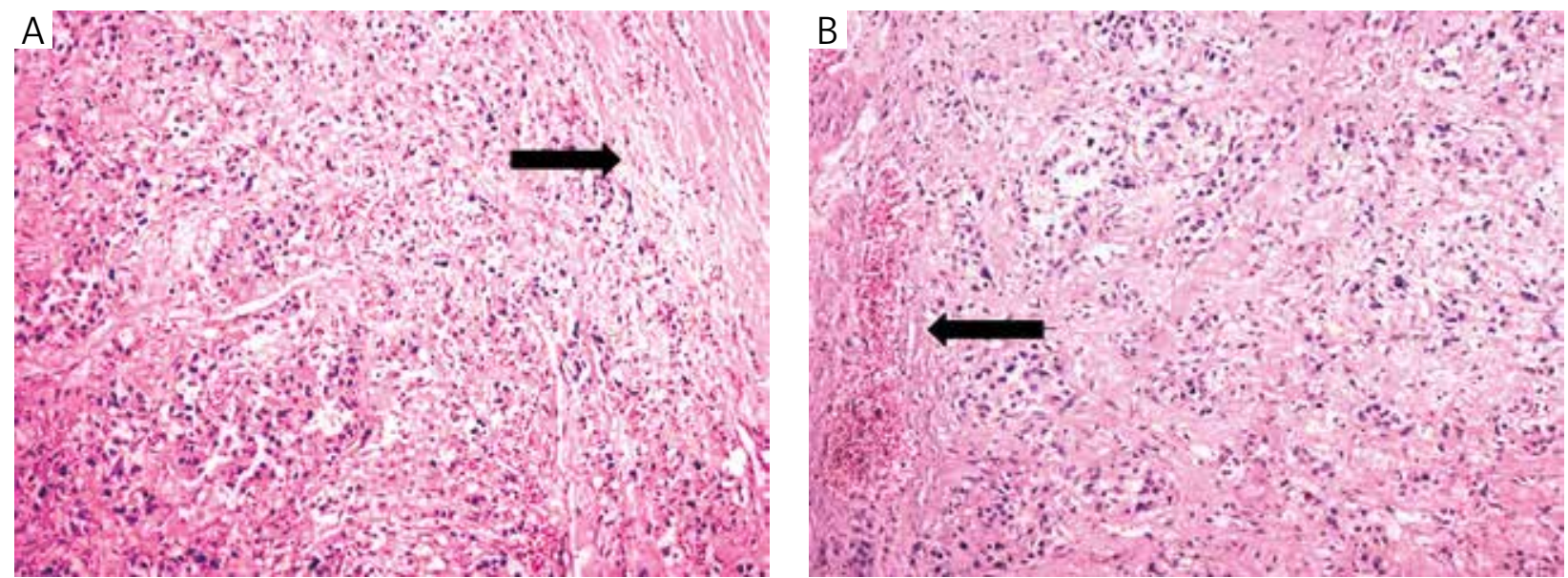

Fig. 3. A) Right side pheochromocytoma specimen presenting encapsulated $(\rightarrow)$ lesion composed of sheets and cords of irregular cells with hyperchromic nucleoli $(\mathrm{HE}$, magnification $100 \times)$. B) Left side pheochromocytoma specimen presenting lesion composed of nests and cords of tumor cells, surrounded by vasculated capsule $(\leftarrow)(\mathrm{HE}$, magnification $100 \times)$

ular hypertrophy and dilatation and atherosclerotic changes were detected in all three coronary arteries, with narrowing up to more than $50 \%$ of the lumens. A $3 \times 2 \times 3 \mathrm{~cm}$ deep-red, loose area surrounded by pale yellow hue was detected in the posterior wall of the left ventricle and posterior portion of the septum, which histologically demonstrated that most of the cardiomyocytes were myocytolytic with marked eosinophilic cytoplasm without nuclei, wavy fibers and marked infiltration of neutrophils, consistent with acute myocardial infarction. Lungs and liver demonstrated marked degrees of congestion. Autopsy findings and histological study excluded any tumors or cystic lesions out of the spectrum of VHL disease in the brain, cerebellum, brain stem, spinal cord, petrous bones, both kidneys, pancreas, and both epididymides. The peripheral sympathetic and parasympathetic chains did not contain any lesions. An autopsy of the eyes was not performed.

Immunohistochemical markers for adrenocortical origin including SF- $1, \mathrm{c} 17$, scc and $3 \beta$-HSD demonstrated positive immunoreactivity in surrounding normal adrenal cortex but negative in tumor cells of both adrenals (Fig. 4A). Neuroendocrine markers such as chromogranin A and synaptophysin were diffusely and intensively positive in both adrenal tumors (Fig. 4B, C). These findings resulted in the diagnosis of bilateral pheochromocytoma. In addition, SDHB immunoreactivity in tumorcells was negative(Fig.4D). This suggested germline mutations either in the $S D H B$ or the $S D H D$ gene. Therefore, genetic studies of these two genes were performed. Once the results were negative, other candidate genes such as RET and $V H L$ were also included and studied. RET exons 10,11 and 16 were negative. Finally, we detected a mutation in the $V H L$ gene located in exon 3: VHL c. $499 \mathrm{C}>\mathrm{T}$. This is a missense mutation and causes an amino acid change (Arg167Trp).

\section{Discussion}

Hereditary pheochromocytoma and paraganglio$\mathrm{ma}$ is a rare disease but still remains a challenging diagnosis, due to its occurrence at young age of presentation and frequently nonspecific clinical symptoms $[1,2]$. Despite improved diagnostic and surgical techniques, there are still a significant number of cases discovered only at the time of autopsy. Some large retrospective studies from North America, Australia, Africa and Asia have demonstrated that 17\% to $23 \%$ of pheochromocytomas were identified only at the time of an autopsy. Of these, $36 \%$ of the patients died because of cardiovascular complications $[10,11]$.

In this particular case, the relatively young age at presentation, multifocal appearance of the tumor and negative SDHB immunohistochemical result suggest more likely the inherited features or genetic background of this patient. Despite all assumptions the genetic investigation did not reveal mutation in the $S D H B$ and $S D H D$ genes. Thus for the approach to a potential hereditary disorder, clinical information was used, namely the young age of 26 and bilateral pheochromocytoma. The European-American-Pheochromocytoma-Paraganglioma Registry based in Freiburg currently (at March 31, 2013) has 272 entries for symptomatic bilateral pheochromocytoma. Of these 76 had VHL, 40 RET, 10 SDHD and 3 SDHB mutation. Therefore we analyzed the RET and $V H L$ genes. Whilst RET mutation screening was negative, VHL analyses revealed a mutation in the VHL gene.

Von Hippel-Lindau disease is characterized by mutations in the $V H L$ gene located in the short arm of chromosome 3, with three exons coding for two isoforms of the VHL protein [1]. The VHL protein is considered to regulate transcription of the hypoxia- 

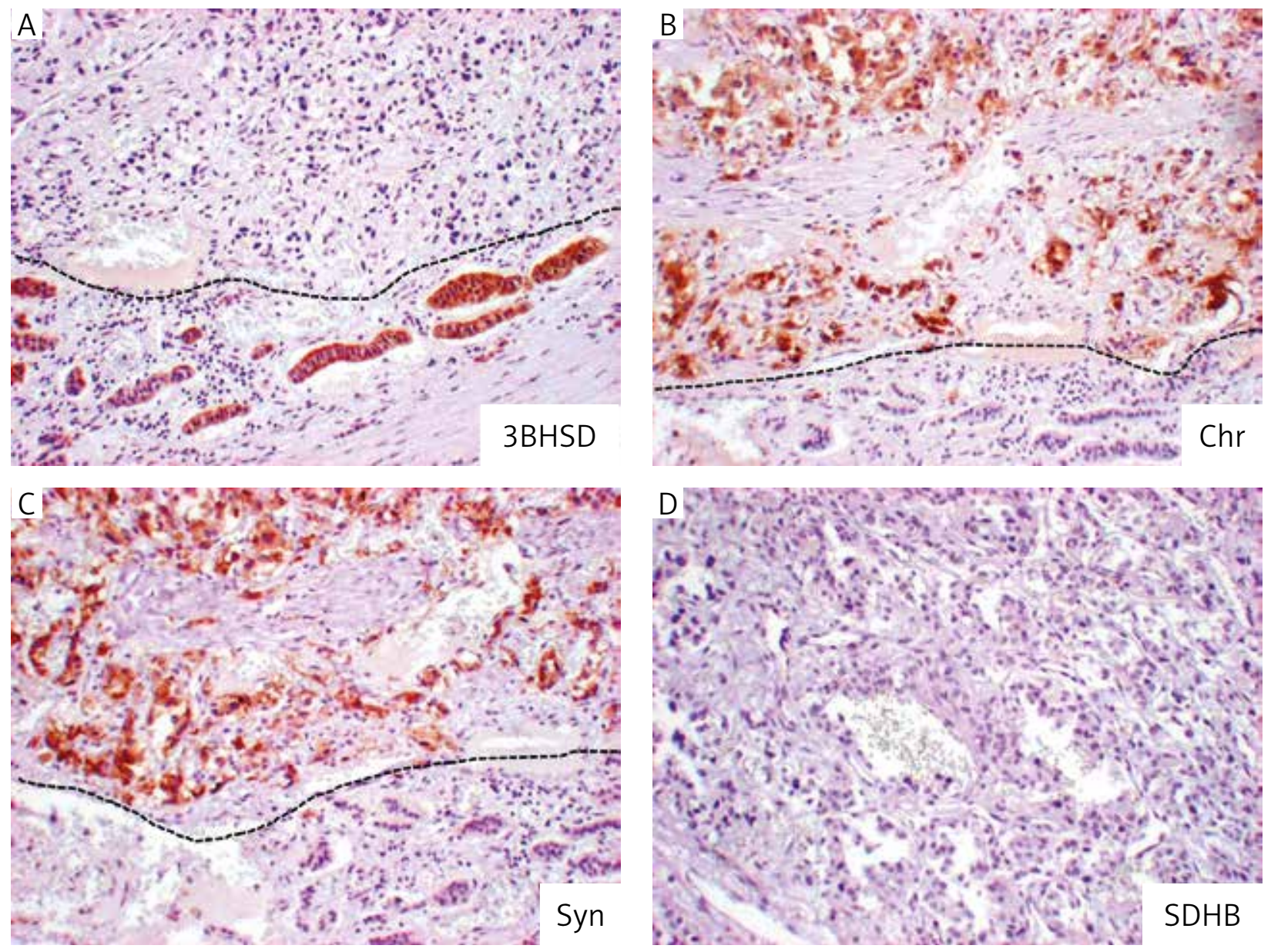

Fig. 4. A) Intensive $3 \beta-H S D$ staining in the cytoplasm of normal corticoadrenal cells (area under the line, magnification $100 \times$ ). B) Intensively positive chromogranin A immunoreactivity of the tumor cells (area above the line, magnification $100 \times$ ). C) Positive synaptophysin staining of the tumor cells (area above the line, magnification $100 \times$ ). D) Absence of SDHB immunoreactivity of the tumor cells (magnification $100 \times$ )

-inducible genes, in particular, hypoxia-inducible factors (HIFs) ubiquitination and proteasome degradation, occurring in normoxic conditions [9, 10]. The mutation in the VHL gene and lack of possibility of HIF degradation in the tumor cells resemble a hypoxia condition [10].

Other pheochromocytoma/paraganglioma associated syndromes are paraganglion syndrome type 1 (PGL1) and type 4 (PGL4). These are caused by mutations in subunits of the succinate dehydrogenase (SDH) complex, respectively in subunit D (SDHD) and subunit $\mathrm{B}$ (SDHB). The SDH complex is involved in the mitochondrial respiratory chain, so is known as mitochondrial II complex, and also plays the role of a component of the Krebs cycle [2]. Recent studies suggested a link between these two processes, scilicet HIF degradation and SDH complex function, respectively, being a possible common origin of the pheochromocytomas in VHL and SDH mutations [11]. The immunohistochemical investigation is presented as a surrogate of these assumptions, showing reduced SDHB and SDHD immunoactivity in all tumors with a SDHB or SDHD mutation and the majority pheochromocytomas with VHL mutation $[11,12]$. The present case appears firstly as an example of absent SDHB immunoactivity in a tumor with a VHL mutation, and secondly as further confirmation of the theory that those pheochromocytomas associated with VHL or SDHB or SDHD mutations possibly have a similar tumorigenesis pathway.

Autbors declare no conflict of interests.

\section{References}

1. McClellan WM, Reiter R, Keiser HR, et al. Clinical and genetic characterization of pheochromocytoma in Von Hippel-Lindau families: comparison with sporadic pheochromocytoma gives insight into natural history of pheochromocytoma. J Urol 1999; 162: 659-664.

2. Lenders J, Eisenhofer G, Mannelli M, Pacak K. Phaeochromocytoma. Lancet 2005; 366: 665-675.

3. Bravo EL, Tagle R. Pheochromocytoma: state-of-the-art and future prospects. Endocr Rev 2003; 24: 539-553.

4. Frankton S, Baithun S, Husain E, et al. Phaeochromocytoma crisis presenting with profound hypoglycaemia and subsequent hypertension. Hormones (Athens) 2009; 8: 65-70. 
5. Jones DJ, Durning P. Phaeochromocytoma presenting as an acute abdomen: report of two cases. Brit Med J 1985; 291: 1267-1268.

6. Steppan J, Shields J, Lebron R. Pheochromocytoma presenting as acute heart failure leading to cardiogenic shock and multiorgan failure. Case Rep Med 2011; doi:10.1155/2011/596354.

7. Cohen CD, Dent DM. Pheochromocytoma and acute cardiovascular death (with special reference to myocardial infarction). Postgrad Med J 1984; 60: 111-115.

8. Lo CY, Lam KY, Wat MS, Lam KS. Adrenal pheochromocytoma remains a frequently overlooked diagnosis. Am J Surg 2000; 179: 212-215.

9. Fishbein L, Nathanson K. Pheochromocytoma and paraganglioma: understanding the complexities of the genetic background. Cancer Genet 2012; 205: 1-11.

10. Lei L, Mason S, Liu D, et al. Hypoxia-inducible factor-dependent degeneration, failure, and malignant transformation of the heart in the absence of the von Hippel-Lindau protein. Mol Cell Biol 2008; 28: 3790-3803.

11. Dahia PL, Ross KN, Wright ME, et al. A HIF1 $\alpha$ regulatory loop links hypoxia and mitochondrial signals in pheochromocytomas. PLoS Genet 2005; 1: 72-80.

12. Pollard PJ, El-Bahrawy M, Poulsom R, et al. Expression if HIF$1 \alpha$ HIF- $2 \alpha$ (EPSA 1 ), and their target genes in paraganglioma and pheochromocytoma with VHL and SDH mutations. J Clin End Metab 2006; 91: 4593-4598.

\section{Address for correspondence}

\section{Svitlana Bachurska}

Department of General and Clinical Pathology

Medical University of Plovdiv

University Hospital "St. George"

bul. "Vasil Aprilov" 15A

4000 Plovdiv, Bulgaria

e-mail: svitba@gmail.com 